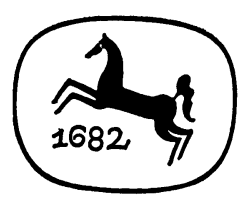

Dynamik semiotischer Systeme 
Winfried Nöth

\section{Dynamik semiotischer Systeme}

Vom altenglischen Zauberspruch

zum illustrierten Werbetext

J. B. Metzlersche Verlagsbuchhandlung Stuttgart 
Meinen Eltern

IN DANKBARKeIT GEWIDMET

Zur Umschlagabbildung: Sie gibt ein Beispiel für den sogenannten geistlichen Bänkelsang, der hier mit dem Devotionalienhandel verbunden ist. Es werden Unglücksfälle erzählt, bei denen die Madonna hilfreich erschien, und gleichzeitig kleine Andachtsbilder mit dem .Gnadenbild der Muttergottes verkauft. Bei der Vorlage handelt es sich um eine kolorierte Kreidelithographie (Anonym; Italien um 1830), die sich in den Beständen der Puppentheatersammlung der Stadt München befindet.

CIP-Kurztitelaufnahme der Deutschen Bibliothek

Nöth, Winfried

Dynamik semiotischer Systeme: vom altengl.

Zauberspruch zum ill. Werbetext. - 1. Aufl. -

Stuttgart: Metzler, 1977.

ISBN 978-3-476-00370-6

ISBN 978-3-476-00370-6

ISBN 978-3-476-03083-2 (eBook)

DOI 10.1007/978-3-476-03083-2

(C) Springer-Verlag GmbH Deutschland 1977

Ursprünglich erschienen bei J. B. Metzlersche Verlagsbuchhandlung

und Carl Ernst Poeschel Verlag GmbH in Stuttgart 1977 


\section{Inhaltsverzeichnis}

Einleitung .................... VII

1. Der semiotische Rabmen der Textanalyse . . . . . . . . . . . . 1

1.1 Das semiotische Spektrum . . . . . . . . . . . . . . 1

1.2 Semiotik in Relation zu anderen Wissenschaften: Der äußere Rahmen der Semiotik . . . . . . . . . . . . . . . 1

1.3 Was ist ein Text? . . . . . . . . . . . . . . . . 2

1.4 Semiotische Textanalyse ............... 3

1.5 Linguistik, Textlinguistik und Semiotik . . . . . . . . . . . . . 3

1.6 Drei semiotische Rahmen der Textanalyse . . . . . . . . . . . 4

1.7 Exkurs: Der semiotische Rahmen poetischer Texte . . . . . . 7

2. Genese und Arbitraritätsgrade des Zeichengebrauchs . . . . . . . . . 12

2.1 Problemstellung und semiotischer Rahmen . . . . . . . . . . . 12

2.2 Arbitrarität der Zeichentypen pragmatisch bestimmt . . . . . . . 14

2.3 Ontogenese des Zeichengebrauchs . . . . . . . . . . . . . . . 16

2.4 Phylogenese des Zeichengebrauchs . . . . . . . . . . . . . . . 19

2.5 Regression des Zeichengebrauchs .............. 24

3. Semiotik des altenglischen Zauberspruchs . . . . . . . . . . . . . . 29

3.1 Problemstellung und semiotischer Rahmen . . . . . . . . . . . 29

3.2 Philologische Analysen der altenglischen Zaubersprüche . . . . . 29

3.3 Merkmale und Definition des altenglischen Zauberspruchs . . . . 30

3.4 Die semantische Dimension des altenglischen Zauberspruchs . . . 35

3.5 Die syntaktische Dimension des altenglischen Zauberspruchs . . . 39

3.6 Die pragmatische Dimension des altenglischen Zauberspruchs . . . 42

3.7 Mangel an Differenzierung als Merkmal des Zauberspruchs . . . 43

3.8 Bedingungen für erfolgreiches Kommunizieren in der Magie . . . 44

3.9 Magische Kommunikation heute . . . . . . . . . . . 45

4. Reklame als primitive Textform . . . . . . . . . . . . . . 47

4.1 Problemstellung . . . . . . . . . . . . . . . . . 47

4.2 Grundlagen der semiotischen Reklameanalyse . . . . . . . . . . 48

4.3 Primitive Argumentationsformen in Reklametexten . . . . . . . 52

4.4 Primitive Argumentationsformen als wirksame Úberzeugungsstrategie 62

5. Prälogik in Reklame und Schizophrenie . . . . . . . . . . . . . 66

5.1 Problemstellung . . . . . . . . . . . . . . . . . . 66

5.2 Struktur der prälogischen und der logischen Schlüsse . . . . . . . 66

5.3 Prälogik in der Reklame . . . . . . . . . . . . . . . 73

5.4 Warum argumentiert Reklame prälogisch? . . . . . . . . 81

6. Dynamische Eigenschaften semiotischer Systeme . . . . . . . . . 83

6.1 Der statische Systembegriff der synchronen Linguistik . . . . . . 83

6.2 Eigenschaften dynamischer Systeme . . . . . . . . . . . . 84

6.3 Ansätze zur Analyse dynamischer Prozesse in der Linguistik . . . . 89

6.4 Die Regelung der sprachlichen Kommunikation . . . . . . . . . 92

6.5 Systemveränderung durch sprachliche Kommunikation . . . . . . 97

7. Fehler im Rabmen einer dynamischen Linguistik . . . . . . . . . . . 99

7.1 Problematisierung . . . . . . . . . . . . . . . . . . . 99

7.2 Die Rolle des Fehlers in Linguistik und Sprachgebrauch . . . . 100 
7.3 Fehler im Sprachgebrauch . . . . . . . . . . . . . . 103

7.4 Spracherwerbsprozesse . . . . . . . . . . . . . . . 106

7.5 Fehler als Kontrollverfahren der Linguistik . . . . . . . . . . 109

8. Textstörung und -rekonstruktion in der Schizophrenie . . . . . . . . 121

8.1 Problemstellung . . . . . . . . . . . . . . . . . 121

8.2 Assoziationsstörungen in der Schizophrenie . . . . . . . . . . . 121

8.3 Restrukturierung der gestörten Textstruktur . . . . . . . . 127

9. Systemstörung und -rekonstruktion in der Diachronie . . . . . . . . 135

9.1 Problemstellung ................... 135

9.2 Sprachwandel als Systemveränderung . . . . . . . . . . . . 137

9.3 Homöostatische Prozesse in der Geschichte der englischen Sprache 138

9.4 Offenheit und Wandel des Sprachsystems . . . . . . . . . . . . 144

9.5 Textdiachronie . . . . . . . . . . . . . . . . . 145

10. Die altenglische Literatur als dynamisches System . . . . . . . . . . 147

10.1 Dynamik literarischer Systeme . . . . . . . . . . . . . . . . 147

10.2 Das Narrative als Paradigma eines dynamischen Textsystems . . . 147

10.3 Textsortenbestimmung auf der Grundlage des Regelkreismodells . 152

10.4 Dynamische Relationen innerhalb eines Textkorpus . . . . . . . 154

10.5 Typologie und Komplementarität altenglischer Texte nach Kriterien der Abbildung . . . . . . . . . . . . . . . . . 155

10.6 Typologie und Komplementarität altenglischer Textsorten nach Kriterien der linguistischen Matrix . . . . . . . . . . . . 154

10.7 Die altenglische Literatur als System . . . . . . . . . . . . . . 162

10.8 Instabilität, Problembewußtsein und Theoriebildung . . . . . . 164

Anmerkungen . . . . . . . . . . . . . . . . 166

Bildnachweis .................... . 181

Literaturverzeichnis . . . . . . . . . . . . . . . . . . 182

Autorenregister . . . . . . . . . . . . . . . 195

Sachregister ... . . . . . . . . . . . . . . 199 


\section{Einleitung}

"Dynamik semiotischer Systeme« verweist auf die zwei theoretischen Bezugsrahmen, die den Sprach- und Textanalysen dieses Buches zugrunde liegen: die Semiotik und die Systemtheorie. - Der Untertitel deutet das breite Spektrum der Analysegegenstände an, das von der altenglischen Sprache und Literatur bis zum illustrierten Werbetext, von der Sprachgenese bis zur Sprachpathologie reicht. Nur scheinbar handelt es sich um ein sehr heterogenes Feld semiotischer Phänomene. Detaillierte Analysen zeigen überraschende Gemeinsamkeiten in der Tiefenstruktur dieser oberflächlich divergierenden semiotischen Phänomene.

Der unifizierende Bezugsrahmen erfordert zunächst die Berücksichtigung des jeweiligen Kontextes: Sprache und Texte werden nicht isoliert, sondern im Rahmen von Situationen, Handlungen und des begleitenden nichtverbalen Zeichengebrauchs analysiert. Die Fruchtbarkeit dieser semiotischen Erweiterung der Analyse zeigt sich besonders in den Untersuchungen zur Poetizität und Sprachgenese, zum Zauberspruch und zum illustrierten Werbetext. Die zweite Komponente des Bezugsrahmens führt zur Analyse der Eigenschaften die die semiotischen Phänomene als offene und dynamische Systeme kennzeichnen. Offenheit ist das Merkmal eines jeden normalen Kommunikationssystems. Die Folgen einer pathologischen Schließung des Kommunikationssystems werden in Texten schizophrener Patienten analysiert. Dabei werden Parallelen zwischen den Stufen der Genese und des Zerfalls semiotischer Systeme sichtbar. Von der Genese über den Zerfall schließt sich der Zyklus der Analyse mit dem Versuch der Rekonstruktion der gestörten semiotischen Systeme. Der Versuch der Rekonstruktion der gestörten Semiose führt nicht nur zu Erkenntnissen über die Struktur pathologischer Phänomene; besonders am Beispiel der Fehlerlinguistik wird sichtbar, wie Fehlleistungen einen Einblick in die Struktur des sprachlichen Normalsystems geben können.

Die Systemeigenschaft der Offenheit und Dynamik zeigt in verschiedenen Graden die Evolution der englischen Sprache und Literatur. Während in der Sprachevolution dynamische Kräfte sichtbar werden, die einer allzu großen Störung des Systems entgegenwirken (Homöostase), zeigt die Evolution literarischer Systeme einen höheren Grad an Offenheit. Ihr Ziel ist die literarische Innovation, die eine permanente Störung und Vergrößerung des Systems (Homöorhese) impliziert.

Offenheit und Dynamik ist schließlich nicht nur ein Phänomen, das im Objektbereich zu beobachten ist; auch für das System der semiotischen Theoriebildung gilt das Postulat der Offenheit und dynamischen Weiterentwicklung der eigenen Untersuchungsmethoden. 